\title{
Innate immune activation of astrocytes impairs neurodevelopment via upregulation of follistatin-like 1 and interferon-induced transmembrane protein 3
}

Shinnosuke Yamada ${ }^{1 \dagger}$, Norimichi Itoh $^{1 \dagger}$, Taku Nagai $^{1 \dagger}$, Tsuyoshi Nakai $^{1}$, Daisuke Ibi ${ }^{2}$, Akira Nakajima $^{3}$, Toshitaka Nabeshima ${ }^{4}$ and Kiyofumi Yamada ${ }^{1 *}$

\begin{abstract}
Background: Polyriboinosinic-polyribocytidylic acid (polyl:C) triggers a strong innate immune response that mimics immune activation by viral infections. Induction of interferon-induced transmembrane protein 3 (Ifitm3) in astrocytes has a crucial role in polyl:C-induced neurodevelopmental abnormalities. Through a quantitative proteomic screen, we previously identified candidate astroglial factors, such as matrix metalloproteinase-3 (Mmp3) and follistatin-like 1 (Fst|1), in polyl:C-induced neurodevelopmental impairment. Here, we characterized the Ifitm3dependent inflammatory processes focusing on astrocyte-derived Fstl1 following polyl:C treatment to assess the neuropathologic role of Fstl1.

Methods: Astrocytes were treated with PBS (control) or polyl:C $(10 \mu \mathrm{g} / \mathrm{mL})$. The conditioned medium was collected $24 \mathrm{~h}$ after the polyl:C treatment and used as astrocyte condition medium (ACM). The expression of Fstl1 mRNA and extracellular Fstl1 protein levels were analyzed by quantitative PCR and western blotting, respectively. For functional studies, neurons were treated with ACM and the effects of ACM on dendritic elongation were assayed. To examine the role of Fstl1, recombinant Fstl1 protein and siRNA for Fstl1 were used. To investigate the expression of Fstl1 in vivo, neonatal mice were treated with vehicle or polyl:C on postnatal day 2 to 6.

Results: ACM prepared with polyl:C (polyl:C ACM) contained significantly higher Fstl1 protein than control ACM, but no increase in Fstl1 was observed in polyl:C ACM derived from Ifitm3-deficient astrocytes. We found that the production of Fstl1 involves the inflammatory responsive molecule Ifitm3 in astrocytes and influences neuronal differentiation. In agreement, the levels of Fstl1 increased in the hippocampus of polyl:C-treated neonatal mice. COS7 cells co-transfected with both Fstl1 and Ifitm3 had higher extracellular levels of Fstl1 than the cells transfected with Fstl1 alone. Treatment of primary cultured hippocampal neurons with recombinant Fstl1 impaired dendritic elongation, and the deleterious effect of polyl:C ACM on dendritic elongation was attenuated by knockdown of Fstl1 in astrocytes.

Conclusions: The extracellular level of Fstl1 is regulated by Ifitm3 in astrocytes, which could be involved in polyl:Cinduced neurodevelopmental impairment.
\end{abstract}

Keywords: Astrocyte, Fstl1, Ifitm3, Immune response, Neuron, polyl:C, Schizophrenia, Viral infection

\footnotetext{
* Correspondence: kyamada@med.nagoya-u.ac.jp

†'Shinnosuke Yamada, Norimichi Itoh and Taku Nagai contributed equally to this work.

${ }^{1}$ Department of Neuropsychopharmacology and Hospital Pharmacy, Nagoya University Graduate School of Medicine, 65 Turumai-cho, Showa-ku, Nagoya, Aichi 466-8560, Japan

Full list of author information is available at the end of the article
}

(c) The Author(s). 2018 Open Access This article is distributed under the terms of the Creative Commons Attribution 4.0 International License (http://creativecommons.org/licenses/by/4.0/), which permits unrestricted use, distribution, and reproduction in any medium, provided you give appropriate credit to the original author(s) and the source, provide a link to the Creative Commons license, and indicate if changes were made. The Creative Commons Public Domain Dedication waiver (http://creativecommons.org/publicdomain/zero/1.0/) applies to the data made available in this article, unless otherwise stated. 


\section{Background}

Immune activation in the CNS is associated with the pathophysiology and/or etiology of psychiatric disorders. For example, inflammatory cytokine levels are altered in the serum of schizophrenia patients [1, 2]. Pro- and anti-inflammatory cytokines in patients with major depressive disorders are inversely correlated with severity and symptoms of major depression [3]. Epidemiological evidence also indicates that the risk of schizophrenia increases in offspring born from mothers infected during pregnancy [4-6]. Although much evidence suggests that reactive astrocytes induced by inflammatory cytokines are involved in neurodevelopmental disorders $[7,8]$, the underlying molecular mechanism is largely unknown.

Interferon-induced transmembrane protein 3 (Ifitm3) is induced by inflammatory cytokines such as interferon- $\beta$, interleukin (IL)-6, and tumor necrosis factor (TNF)- $\alpha$. Ifitm 3 acts as an antiviral factor by restricting virus entry [9-12]. Recent studies have indicated that Ifitm3 expression is increased in patients with schizophrenia [13-15]. Previously, we have reported that Ifitm3 expression in astrocytes is associated with neurodevelopmental impairment and contributes to brain dysfunction in mice that received daily polyI:C injection from postnatal day 2 to day 6 [16]. We also found that humoral factors released from polyI:C-treated astrocytes have crucial roles in Ifitm3-dependent impairment of neuronal maturation of cultured neurons [17]. For instance, matrix metalloprotease-3 (Mmp3) is released by polyI:C-treated astrocytes and impairs neuronal maturation [18]. In addition to Mmp3, we have identified several candidates of humoral factors including follistatin like-1 (Fstl1) by proteomic analysis [18].

To investigate the regulation of Fstl1 production and release, we monitored extracellular levels of Fstl1 protein in astrocyte cultures, which were stimulated with polyI:C or prepared from Ifitm3 knockout (KO) mice. We found that an immune activation induced by polyI:C upregulates Fstl1 mRNA expression in astrocytes, and that polyI:C-treated astrocytes have increased extracellular protein levels of Fstl1. Notably, in astrocytes from Ifitm3 $\mathrm{KO}$ mice, polyI: $\mathrm{C}$ treatment failed to increase the extracellular Fstl1 level. We also demonstrated that recombinant Fstl1 impaired dendritic neurite outgrowth of cultured hippocampal neurons, but knockdown of Fstl1 partially rescued polyI:C-induced impairment of neurite elongation. These results suggest that Fstl1 has an important role in Ifitm3-dependent neuronal impairment.

\section{Methods}

\section{Animals}

C57BL/6J mice were purchased from Japan SLC Inc. (Hamamatsu, Japan). Homozygous Ifitm3 $^{-/-}$[Ifitm3 KO] mice were generated and characterized as described previously [19]. The animals had free access to food (CE-2, Clea Japan, Tokyo, Japan) and water and were kept under controlled conditions $\left(23 \pm 1{ }^{\circ} \mathrm{C}\right)$ with a constant light-dark cycle (light 9:00-21:00). All animals were handled in accordance with the guidelines established by the Institutional Animal Care and Use Committee of Nagoya University, the Guiding Principles for the Care and Use of Laboratory Animals approved by the Japanese Pharmacological Society, and the National Institutes of Health Guide for the Care and Use of Laboratory Animals.

\section{Astrocyte culture and astrocyte-conditioned medium (ACM) preparation}

Secondary astrocyte cultures were prepared as described previously [18]. Briefly, cortices and hippocampi of neonatal mice at postnatal day (PD) 1-2 were mechanically dissociated and digested with $0.3 \%$ dispase (Roche Diagnostics $\mathrm{GmbH}$, Mannheim, Germany) and 0.4\% DNase (Roche Diagnostics $\mathrm{GmbH}$ ). The cells were suspended in Dulbecco's modified Eagle's medium (DMEM, Sigma-Aldrich, St. Louis, MO) containing 10\% fetal bovine serum (FBS, Gibco-BRL, Gaithersburg, MD) and filtered through a $40 \mu \mathrm{m}$ nylon cell strainer (Falcon-Becton Dickinson, Le Pont de Claix, France). The cell suspension was cultured in a T150 flask at a density of six neonate brains per flask at $37{ }^{\circ} \mathrm{C}$ with $5 \% \mathrm{CO}_{2}$. Confluent primary astrocyte cultures were purified by shaking, plated onto 6-well plates, and grown to confluence in all experiments. Under these conditions, more than $95 \%$ of cells were glial fibrillary acidic protein (GFAP)-positive (a marker for astrocytes) and negative for tau/MAP2 and CD11b (neuronal and microglial markers, respectively). Culture medium was replaced with Neurobasal Medium (Invitrogen, Eugene, OR) supplemented with B-27 (Invitrogen) and $1 \mathrm{mM}$ glutamine (Sigma-Aldrich) 6 days before treatment with polyI:C (Sigma-Aldrich). This medium was replaced 3 days before and $24 \mathrm{~h}$ before treatment with polyI:C. For western blotting, B-27 was excluded from the last medium. Astrocytes were treated with PBS (control) or polyI:C $(10 \mu \mathrm{g} / \mathrm{mL})$, and conditioned medium was collected $24 \mathrm{~h}$ after the polyl:C treatment. For the time course analysis, conditioned medium was collected $6 \mathrm{~h}$ and $12 \mathrm{~h}$ after polyI:C treatment. Conditioned media were centrifuged at $1000 \times g$ for $10 \mathrm{~min}$ at $4{ }^{\circ} \mathrm{C}$, and the supernatants were used as ACM.

\section{Microglia culture and microglia-conditioned medium (MCM) preparation}

2 weeks after seeding primary astrocyte cultures, microglia were separated from the underlying astrocytic monolayer by shaking for $3 \mathrm{~h}$ at $150 \mathrm{rpm}$. The supernatants, including floating microglia, were centrifuged at $1000 \mathrm{rpm}$ for $10 \mathrm{~min}$ and the pellet was resuspended in 
fresh culture medium. Microglia were plated at $1 \times 10^{6}$ cells/well in a 6-well plate and culture medium was replaced with neurobasal medium supplemented with $1 \mathrm{mM}$ glutamine $1 \mathrm{~h}$ after plating. The microglial cultures were $>98 \%$ pure as assessed by immunocytochemistry with an anti-Ibal antibody (Wako, Osaka, Japan) in combination with an anti-GFAP antibody (Sigma-Aldrich) as markers for microglia and astrocytes, respectively. PBS (control) or polyI:C $(10 \mu \mathrm{g} / \mathrm{mL})$ were added $24 \mathrm{~h}$ after medium change, and conditioned media were collected $24 \mathrm{~h}$ after polyI:C treatment. Conditioned media were centrifuged at $1000 \times g$ for $10 \mathrm{~min}$ at $4{ }^{\circ} \mathrm{C}$, and the supernatants were used as MCM.

\section{Fstl1 and Ifitm3 transfection for COS7 cells}

Myc-tagged mouse Fstll expression vector was purchased from OriGene Technologies (Rockville, MD), and myc-tagged mouse Ifitm3 vector was generated as described previously [17]. Control (mock) vector was generated by the removal of cDNA sequence from the cloning site of the expression vector using the appropriate restriction enzyme, then blunt-ended and ligated. The day before transfection, trypsinized COS7 cells were plated at $40 \times 10^{4}$ cells per well (6-well plate). Each well was transfected with $1.25 \mu \mathrm{g}$ myc-tagged mouse Ifitm3 and Fstl1 or control vectors using Lipofectamine LTX (Invitrogen) and Opti-MEM I Reduced Serum Medium (Invitrogen). $6 \mathrm{~h}$ after transfection, growth medium was replaced with $2 \mathrm{~mL}$ of MEM without FBS and antibiotics. Conditioned medium and cell lysate samples for western blotting were prepared $30 \mathrm{~h}$ after transfection as described in the "Astrocyte culture and astrocyte-conditioned medium (ACM) preparation" section. Recombinant mouse Fstl1 (rmFstl1) was prepared from conditioned medium after transfection of $2.5 \mu \mathrm{g}$ myc-tagged mouse Fstl1 expression vector. $30 \mathrm{~h}$ after transfection, secreted Fstl1 was purified using c-myc tagged Protein Mild Purification Kit ver.2 (MBL, Nagoya, Japan) according to the manufacturer's instructions. Protein concentration was determined using a protein assay kit (Bio-Rad Laboratories, Hercules, CA, USA).

\section{Primary cultured neurons and ACM treatment}

Primary cultured hippocampal neurons were prepared from C57BL/6J mice on gestational day $15-16$ as described previously [18]. Briefly, embryo hippocampi were trypsinized (with $0.25 \%$ trypsin and $0.01 \%$ DNase) followed by trituration and seeded on coverslips precoated with $0.1 \mathrm{mg} / \mathrm{mL}$ poly-D-lysine at a low density $\left(1.0 \times 10^{4}\right.$ cells/well in a 24 -well plate). Cells were cultured in Neurobasal Medium with B-27 and $1 \mathrm{mM}$ glutamine. The medium was replaced with polyI:C ACM or control ACM and supplemented with $0.75 \mu \mathrm{m}$ cytosine $\beta$-D-arabinofuranoside (Ara-C, Sigma-Aldrich) on DIV2.
For functional studies, rmFstl1 was added to control ACM or culture medium (Neurobasal Medium supplemented with B-27 and $1 \mathrm{mM}$ glutamine) at the final concentrations of $10 \mathrm{nM}, 50 \mathrm{nM}, 100 \mathrm{nM}$, or $300 \mathrm{nM}$ on DIV2. The effects of ACM on dendritic elongation were assayed on DIV7. More than 99\% pure neurons, as evaluated by anti-tau or anti-MAP2 immunostaining, were obtained from this preparation.

\section{Knockdown assay}

siRNA transfection was performed $6 \mathrm{~h}$ before the last medium change. Astrocytes were transfected with Stealth siRNA for Fstl1 (\#1 sense: GCCCAGUUGUCUGC UAUCAAGCUAA, \#1 antisense: UUAGCUUGAUAGCA GACAACUGGGC; \#2 sense: CCUAGACAAGUACU UUAAGAGCUUU, \#2 antisense: AAAGCUCUUAAAGU ACUUGUCUAGG) or Stealth RNAi siRNA negative control (control siRNA) using Lipofectamine RNAiMAX transfection reagent (all from Invitrogen).

\section{Western blotting}

ACM and MCM were concentrated using Vivaspin 2 Hydrosart 5000 MWCO (Sartorius Stedim Biotech $\mathrm{GmbH}$ ). After removing the conditioned medium, the remaining cells were washed with ice-cold PBS and collected in lysis buffer $[20 \mathrm{mM}$ Tris- $\mathrm{HCl}$ ( $\mathrm{pH}$ 7.4), $150 \mathrm{mM} \mathrm{NaCl}, 50 \mathrm{mM} \mathrm{NaF}, 2 \mathrm{mM}$ EDTA, $1 \%$ Triton $\mathrm{X}-100,1 \mathrm{mM}$ sodium orthovanadate, $0.1 \%$ SDS, $1 \%$ sodium deoxycholate and protease inhibitor cocktail (Sigma-Aldrich)]. Protein lysates were centrifuged at $15,000 \times g$ for $20 \mathrm{~min}$. ACM or cell lysates were denatured in Laemmli sample buffer containing 20\% $\beta$-mercaptoethanol at $95{ }^{\circ} \mathrm{C}$ for $5 \mathrm{~min}$. An equal amount of protein for each sample was separated by $10 \%$ SDS-PAGE and transferred to a polyvinylidene fluoride (PVDF) membrane (Millipore). The membrane was blocked with detector block solution (KPL, Gaithersburg, MD). The membrane was incubated with goat anti-Fstl1 antibody (R\&D Systems, Minneapolis, MN) or goat anti-actin antibody (Santa Cruz Biotechnology, Santa Cruz, CA) at $4{ }^{\circ} \mathrm{C}$ overnight. After incubation with horseradish peroxidase-conjugated secondary anti-goat antibody (R\&D Systems) for $2 \mathrm{~h}$, the membrane was incubated with ECL prime western blotting detection reagents (GE Healthcare) and protein bands were detected using a luminescent image analyzer (Atto, Tokyo, Japan).

\section{Total RNA isolation and real-time RT-PCR}

After removing the conditioned medium, total RNA of astrocytes and microglia were prepared using RNeasy Mini Kit (Qiagen, Hilden, Germany) and converted into complementary DNA (cDNA) using the SuperScript III First-Strand Synthesis Kit (Invitrogen). Quantitative real-time PCR was performed on a 7300 Real-Time PCR 
System (Applied Biosystems, Foster City, CA) using Power SYBR Green Master Mix (Applied Biosystems) according to the manufacturer's protocol. The primers used were as follows: forward, GCCTATGCCTACTCCGTGAAGT and reverse, GCCTGGGCTCCAGTCACAT for Ifitm3; forward, CACCAGGGCACAGCAGAAA and reverse, GTGCTCTGTGCCTCTTCTTAGATCT for Fstl1; and forward, CGATGCCCTGAGGCTCTTT and reverse, TGGATGCCACAGGATTCCA for $\beta$-actin used as an internal control. Real-time PCR reactions were conducted as follows: initial $2 \mathrm{~min}$ incubation at $50^{\circ} \mathrm{C}$ and $10 \mathrm{~min}$ incubation at $95{ }^{\circ} \mathrm{C}$, followed by 40 reaction cycles of $95{ }^{\circ} \mathrm{C}$ for $15 \mathrm{~s}$ and $60{ }^{\circ} \mathrm{C}$ for $1 \mathrm{~min}$. Fluorescent signals were monitored at the extension step of $60^{\circ} \mathrm{C}$ in each cycle. For each sample test, each PCR reaction had two replicates and the relative gene expression differences were quantified using the comparative $\mathrm{Ct}$ method $\left({ }^{\Delta \Delta} \mathrm{Ct}\right)$.

\section{Immunocytochemistry}

Cells were fixed in $4 \%$ paraformaldehyde in $0.1 \mathrm{M}$ phosphate buffer ( $\mathrm{pH} 7.4)$ for $20 \mathrm{~min}$ and then permeabilized with $0.1 \%$ Triton X-100 for $10 \mathrm{~min}$. After incubation in blocking solution (1\% goat and $1 \%$ donkey serum in PBS) for 30 min, mouse anti-tau (1:500, Santa Cruz Biotechnology) and rabbit anti-MAP2 (1:1000, Millipore) antibodies diluted in blocking solution were added to the cells. After overnight incubation with primary antibodies at $4{ }^{\circ} \mathrm{C}$, the cells were treated with goat anti-mouse Alexa Fluor (AF) 488 and anti-rabbit AF568 antibodies (1:1,000, Invitrogen) for $2 \mathrm{~h}$ at room temperature. The cells were mounted in fluorescence mounting medium (Dako, Glostrup, Denmark) and photographed under a fluorescence microscope (Zeiss, Jena, Germany) using AxioCam MRc5 (Zeiss).

\section{Dendritic elongation assay}

Dendritic elongation of cultured hippocampal neurons was analyzed in accordance with a previous study [18]. Axons were identified by double immunostaining in terms of tau-positive (axonal marker) and MAP2-negative (dendritic marker), and only MAP2-positive neurites were defined as dendrites. Neurons that clearly had tau- or MAP2-positive neurites were selected randomly by an expert researcher who was blinded to the experimental groups. Dendrites were traced automatically with the same configuration using Neurolucida software (MicroBrightField, Williston, VT) and total dendritic length in a single neuron was calculated using Neuroexplorer (MicroBrightField). This assay was performed for three independent experiments.

\section{Immunohistochemistry (IHC) for polyl:C-treated neonatal mice}

All litters from C57BL/6J mice were randomly divided into two groups: vehicle- and polyI:C-treated. Neonatal C57BL/6J mice were administered with a daily subcutaneous injection of saline (control) or polyI:C (5 mg/kg, Sigma-Aldrich) between PD2 and PD6. Immunohistochemistry was conducted as described previously [17]. Neonatal mice were deeply anesthetized with diethyl ether $24 \mathrm{~h}$ after the final polyI:C treatment and perfused transcardially with saline, followed by $4 \%$ paraformaldehyde in phosphate-buffered saline (PBS, $\mathrm{pH}$ 7.4). The brains were removed and cryoprotected. $20-\mu \mathrm{m}$-thick coronal brain sections were cut on a cryostat and mounted on slides. The sections were denatured in a microwave oven in $0.01 \mathrm{M}$ citrate buffer (pH 6.0). After blocking with $5 \%$ donkey and $5 \%$ goat serum/PBS, mouse anti-glial fibrillary acidic protein (GFAP, a marker for astrocytes, 1:1,000, Sigma-Aldrich) and rat anti-Fstl1 (1:100, R\&D systems) were added to the sections. After washing in PBS, goat anti-mouse Alexa Fluor (AF) 568 and anti-rat AF488 antibodies (1:1,000, Invitrogen) were added to the sections. The samples were observed using a confocal-laser scanning microscope (LSM 700 Axio Imager; Zeiss).

\section{Statistical analysis}

Data are shown as the mean \pm SE. Differences between two groups were analyzed by two-tailed Student's $t$ test and the data distribution was tested for normality with Shapiro-Wilk test. One-way and two-way analyses of variance (ANOVA) followed by Bonferroni post hoc test was applied for differences in three or more groups.

\section{Results}

We previously reported that Fstl1 was a candidate molecule responsible for polyI:C-induced neurodevelopmental impairment, and that depletion of Ifitm3 in astrocytes attenuated polyI:C ACM-induced neurodevelopmental impairment $[17,18]$. We compared changes in Fstl1 protein levels between WT and Ifitm3 $\mathrm{KO}$ astrocytes after polyI:C treatment (Fig. 1a). Two-way ANOVA revealed significant main effects of polyI:C treatment on Fstl1 protein levels in both cell lysates (polyl:C treatment: $F(1,8)=28.80, p<0.01$; genotype: $F(1,8)=0.22$, $p=0.65$; interaction of polyI:C treatment and genotype: $F(1,8)=0.01, p=0.94$, Fig. $1 \mathrm{~b}$ ) and ACM (polyI:C treatment: $F(1,8)=66.09, p<0.01$; genotype: $F(1,8)=37.21$, $p<0.01)$; with an interaction of polyI:C treatment and genotype: $(F(1,8)=23.77, p<0.01$, Fig. 1c). PolyI:C treatment significantly increased Fstl1 protein levels in the cell lysates of both WT and Ifitm3 KO astrocytes $(p<0.05)$ (Fig. 1b). Notably, polyI:C treatment significantly increased Fstl1 levels in ACM from WT astrocytes but had no effect on extracellular Fstl1 levels in ACM from Ifitm3 $\mathrm{KO}$ astrocytes (Fig. 1c). To examine the influence of Ifitm3 expression on extracellular Fstl1 levels, COS7 cells were co-transfected with Fstl1 and Ifitm3. Fstl1 in COS7 cell lysates was not affected by 

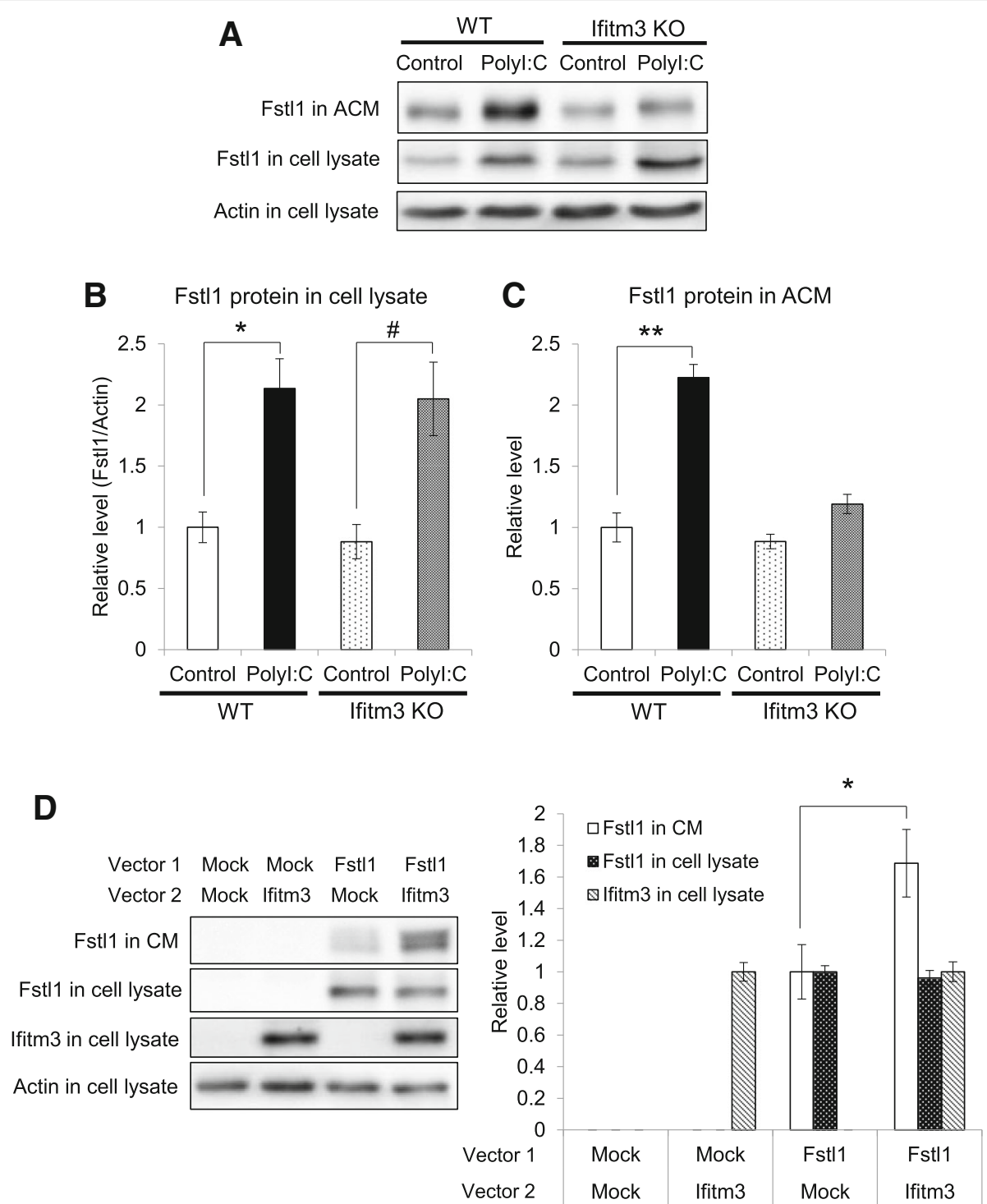

Fig. 1 Ifitm3 regulates the extracellular level of Fstl1. a Representative western blot images of Fstl1 protein expression in cell lysates and ACM derived from WT and Ifitm3 KO astrocytes $24 \mathrm{~h}$ after polyl:C treatment. b, c Fstl1 protein levels in cell lysates (b) and ACM (c) derived from WT and Ifitm3 KO astrocytes after polyl:C treatment. Values indicate the means \pm SE $(n=3) .{ }^{*} p<0.05,{ }^{*} p<0.05$, and ${ }^{* *} p<0.01$ versus the respective control treatment. d The extracellular level of Fstl1 in conditioned medium after Fstl1 and Ifitm3 overexpression. COS7 cells were co-transfected with Fstl1, Ifitm3, and control vectors (mock) as indicated respectively. Overexpression of Ifitm3 increased Fstl1 protein in CM without affecting Fstl1 expression in COS7 cell lysates. Data show the mean $\pm \mathrm{SE}(n=6) .{ }^{*} p<0.05$ versus Fst11/mock. CM, conditioned medium

overexpression of Ifitm3. On the other hand, Fstl1 levels in conditioned medium (CM) from COS7 cells co-transfected with Fstl1 and Ifitm3 were markedly higher than the levels from Fstl1 alone transfection ( $p<0.05$, Fig. 1d). These results suggest that the Fstl1 release is regulated by Ifitm3.

Because microglia are immunocompetent cells in the CNS, it is possible that microglia also play a similar role in the polyI:C-triggered glial inflammatory response. Accordingly, we examined the mRNA expression of Fstl1 and Ifitm3 in astrocytes and microglia. A two-way ANOVA revealed significant effects of polyI:C treatment on Ifitm3 mRNA levels in astrocyte and microglia cell lysates
(polyl:C treatment: $F(1,12)=188.0, p<0.01$; cell type: $F(1,12)=19.91, p<0.01$; interaction of polyI:C treatment and cell type: $F(1,12)=0.36, p=0.56$, Fig. $2 \mathrm{a}$ ). In contrast, there were significant interactions between cell type and polyI:C treatment on Fstl1 mRNA levels (polyI:C treatment: $F(1,12)=25.84, p<0.01$; cell type: $F(1,12)=127.8$, $p<0.01$; interaction of polyI:C treatment and cell type: $F(1,12)=28.04, p<0.01$, Fig. 2b). A multiple-comparison test with Bonferroni post hoc tests indicated that polyI:C treatment induced the expression of Fstl1 mRNA in astrocytes, but not in microglia ( $p<0.01$, Fig. 2b). Furthermore, Fstl1 protein was not detected in MCM with or without 

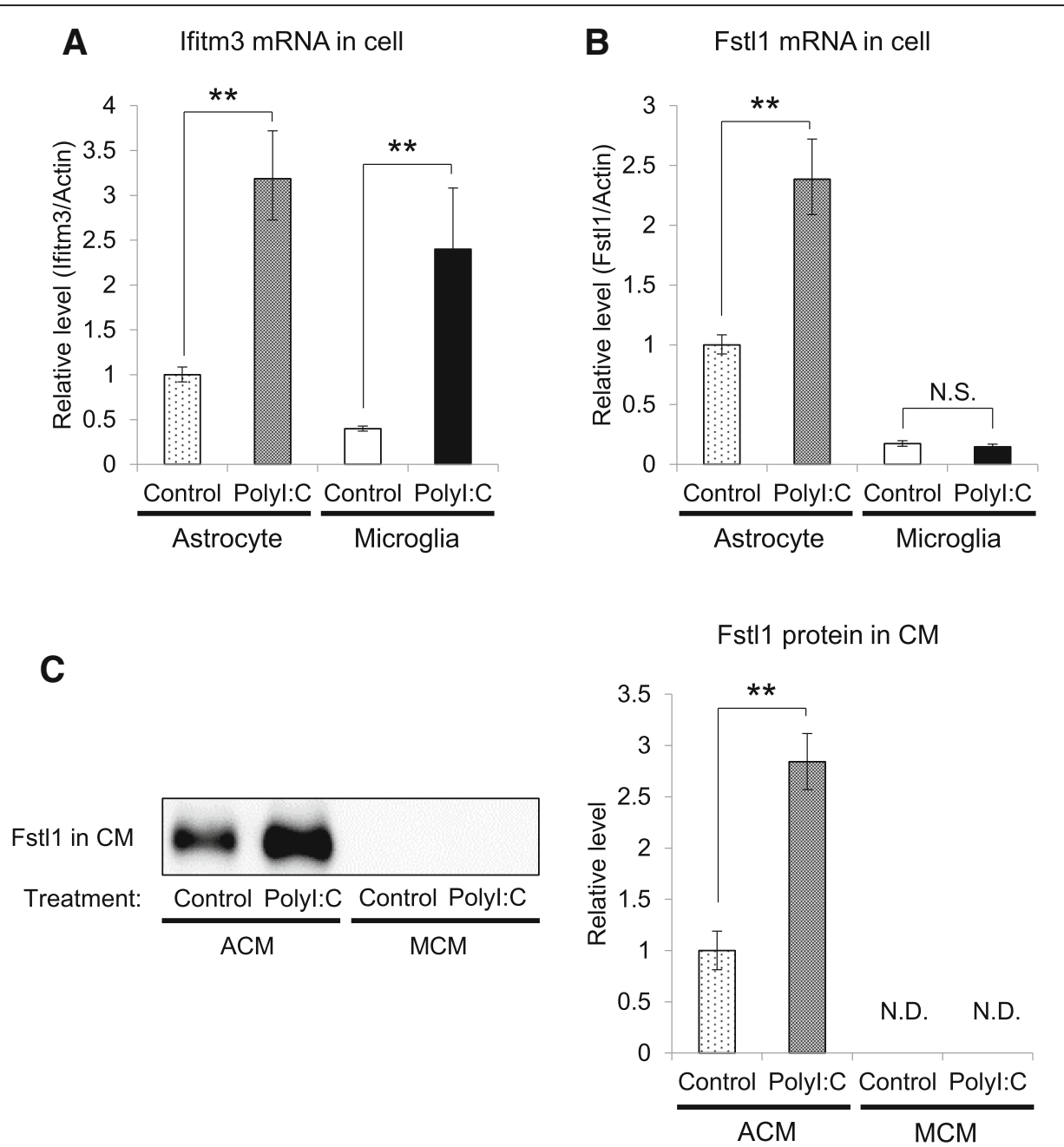

Fig. 2 Changes in Ifitm3 and Fstl1 expression in polyl:C-treated astrocytes and microglia. a Ifitm3 mRNA levels in polyl:C-treated astrocytes and microglia. Values indicate the means $\pm \mathrm{SE}(n=4) .{ }^{* *} p<0.01$ versus the respective control treatment. $\mathbf{b}$ Fstl1 $\mathrm{mRNA}$ levels in polyl:C-treated astrocytes and microglia. Values indicate the means \pm SE $(n=4)$. N.S., not significant. ${ }^{*} p<0.01$ versus control treatment. c Fstl1 protein levels in polyl:C ACM and polyl:C MCM. Values indicate the means \pm SE $(n=7) .{ }^{* *} p<0.01$ versus control. ACM, astrocyte conditioned medium; MCM, microglia conditioned medium. N.D., not detectable

polyI:C treatment, while Fstl1 protein levels were significantly increased in polyl:C ACM compared to that of the control $(p<0.01$, Fig. 2c). These results suggest that Fstl1 is induced by polyI:C treatment in astrocytes but not in microglia, and thereby microglia may not play a role in the regulation of extracellular Fstl1 level.

We previously found that polyI:C-treated ACM impairs dendritic elongation of cultured hippocampal neurons [17]. Therefore, the role of Fstl1 in dendritic elongation was assessed using an RNA interference method. The effect of siRNA was confirmed by western blotting. When primary cultured astrocytes were transfected with control, Fstl1 \#1 or \#2 siRNA, the expression levels of Fstl1 in \#1 or \#2 siRNA were significantly decreased to $34 \%$ and $22 \%$ of control siRNA transfected cell (Additional file 1: Figure S1). Under this condition,
Fstl1 protein levels in ACM were significantly increased by polyI:C treatment $(F(3,12)=14.09, p<0.01$, Fig. 3a). When astrocytes were transfected with either Fstl1 siRNA \#1 or \#2 to down-regulate Fstl1 expression, polyI:C failed to increase extracellular Fstl1 protein levels in ACM, and the levels were similar to those in control ACM $(F(3,12)=14.09, p<0.01$, Fig. 3a). To assess whether Fstl1 plays a negative role in neurite elongation, we measured dendrite length of primary cultured hippocampal neurons which were cultured with the ACM derived from either control or siRNA-treated astrocytes. PolyI:C-treated ACM impaired the dendritic elongation of cultured hippocampal neurons in control siRNA-transfected groups. This impairment was partially attenuated when astrocytes were transfected with either Fstl1 siRNA \#1 or \#2 $(F(3,174)=92.53, p<0.01$, Fig. $3 b)$. 


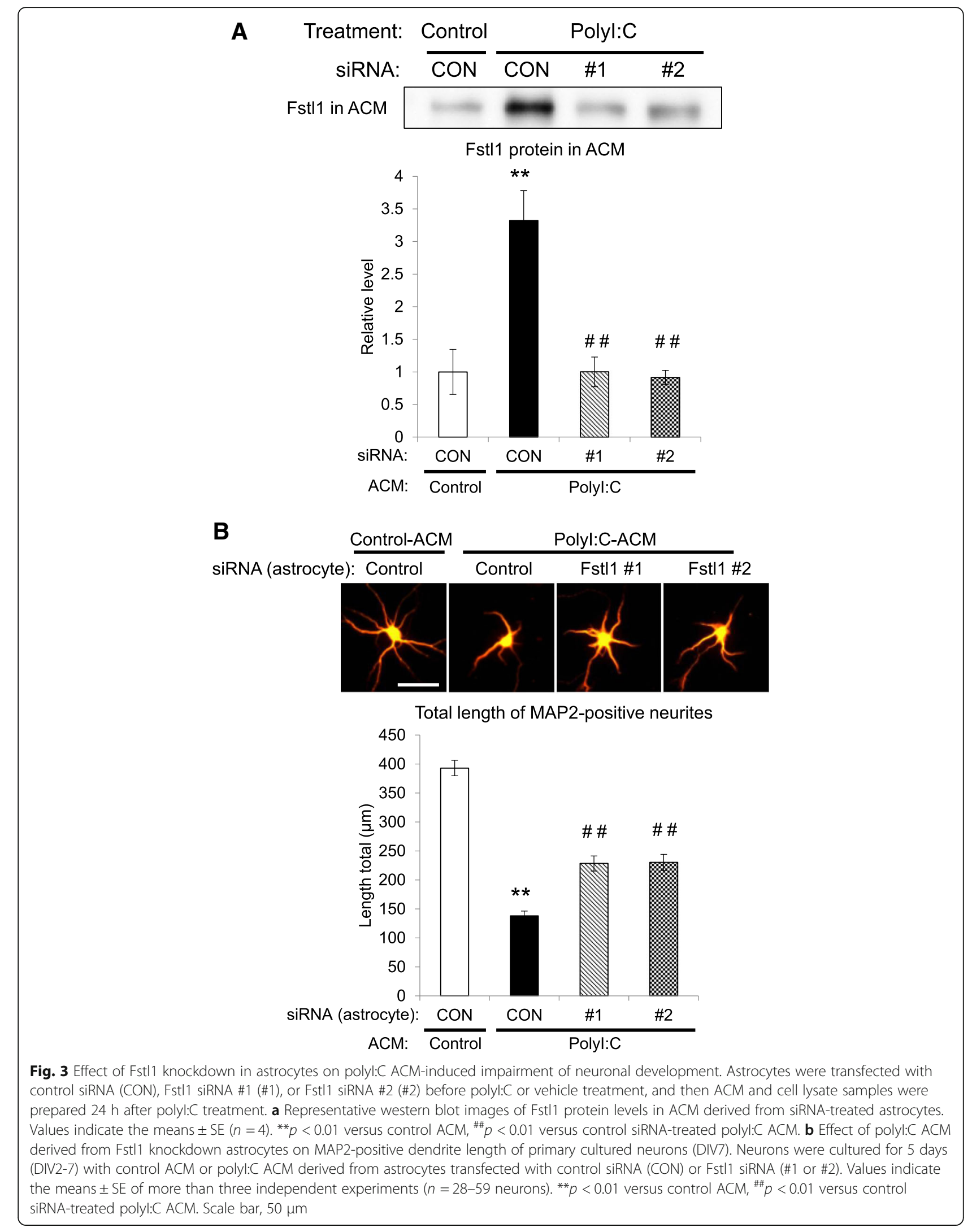


We further examined whether Fstl1 protein could mimic the above deleterious effect of polyI:C ACM. Addition of recombinant mouse Fstl1 protein to control ACM on DIV2 resulted in a concentration-dependent decrease in dendrite length of primary cultured neurons assayed on DIV7 $(F(3,203)=22.08, p<0.01$, Fig. 4a). The decrease in dendrite length induced by rmFstl1 treatment was $12 \%$ (50 nM), 32\% (100 nM), and 35\% (300 $\mathrm{nM}$ ) compared to that treated with control ACM alone, and a significant decrease of dendritic length was observed at more than $100 \mathrm{nM} \mathrm{rmFstl1}(p<0.01$, Fig. 4a). Of note, the addition of $300 \mathrm{nM}$ rmFstl1 to normal culture medium on DIV2 had no effect on dendritic elongation of primary cultured hippocampal neurons assayed on DIV7 (Fig. 4b). rmFstl1 treatment had no effect on branched number of neurites and viability of neurons (Additional files 2 and 3: Figures S2 and S3). These results suggest that Fstl1 itself may not directly inhibit the dendritic elongation of neurons but interrupt neurite elongation by cooperating with some factors in ACM.

Finally, the in vivo expression of Fstl1 in the hippocampus $24 \mathrm{~h}$ after neonatal polyI:C treatment was analyzed by IHC. The expression of Fstl1 was hardly detected in vehicle-treated control mice under the same experimental conditions (Fig. 5a). Fstl1 immunofluorescence was clearly observed in the hippocampus of polyI:C-treated mice, and the signal coincided with GFAP, a marker for astrocytes (Fig. 5a). Approximately, 60\% of astrocytes were positive for Fstl1 in the hippocampus of polyI:C-treated mice
(Fig. 5b). The co-expression of Fstl1 with Iftim 3 in astrocyte was observed in the hippocampus of polyl:C-treated neonatal mice (Additional file 4: Figure S4). The expression level of Fstl1 in polyI:C-treated Ifitm3 $\mathrm{KO}$ mice was comparable to the level in polyI:C-treated WT mice (Additional file 5: Figure S5).

\section{Discussion}

We have previously demonstrated that mice received neonatal polyl:C treatment exhibit some characteristics of neurodevelopmental disorders including cognitive and emotional impairments in adulthood, which is accompanied by the decrease in spine density and dendrite complexity of pyramidal neurons in the frontal cortex $[16,17,20]$. PolyI:C treatment induces expression of several inflammation-related genes, and induction of these molecules plays a pivotal role in polyI:C-induced neuronal impairment. We have already demonstrated that Ifitm3 expression is increased by polyI:C treatment in astrocytes. Ifitm 3 protein localizes to the early endosomes and reduces the endocytic activity of astrocytes, which may change the composition of the extracellular humoral factors. Ifitm3-mediated accumulation of humoral factors released from astrocytes is a determinant in polyI:C-induced neurodevelopmental impairment [17]. Mmp-3 is one of such humoral factors that contribute to the polyI:C-induced neuronal impairment [18].

In the present study, we demonstrated that extracellular Fstl1 level was also dramatically increased in

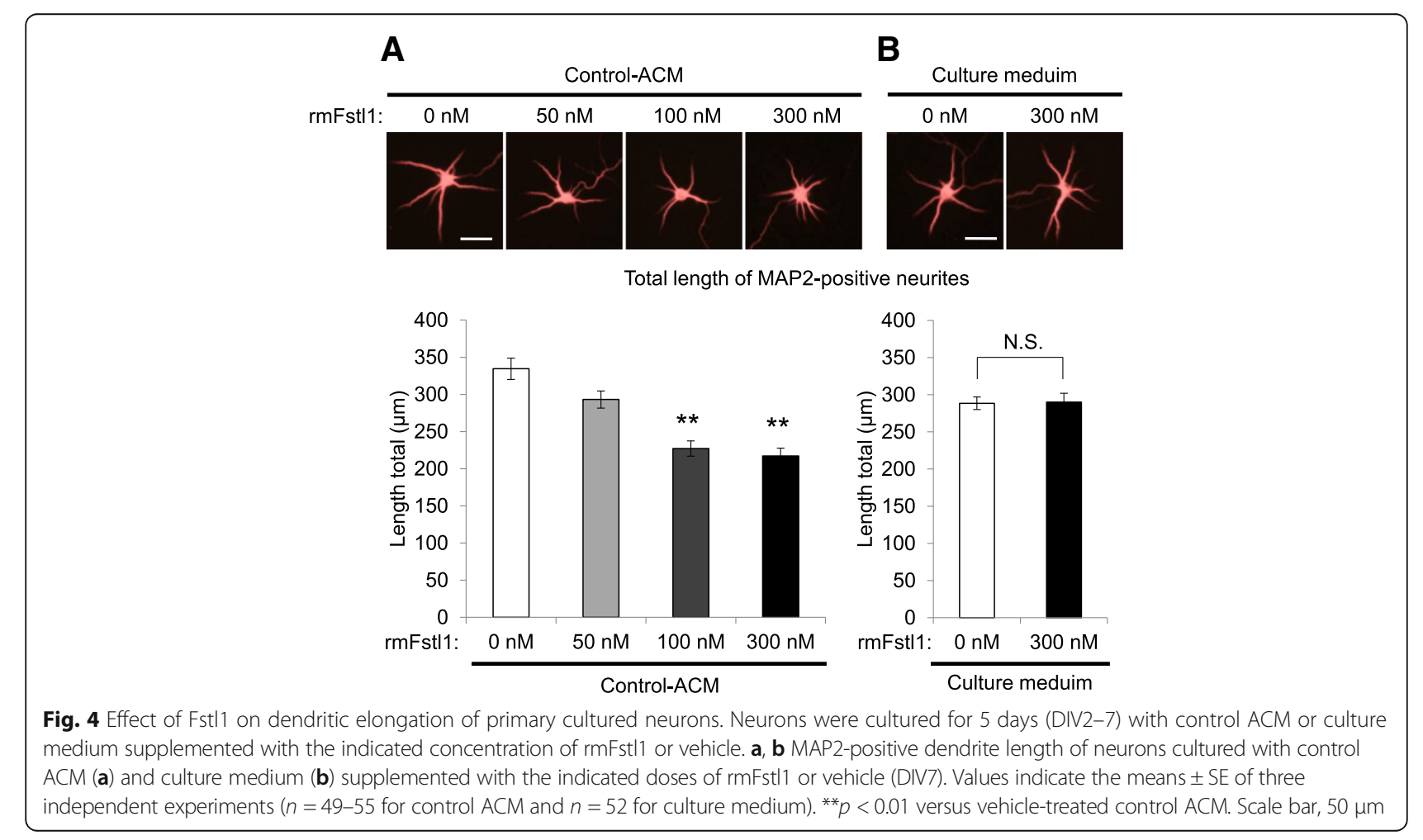


A

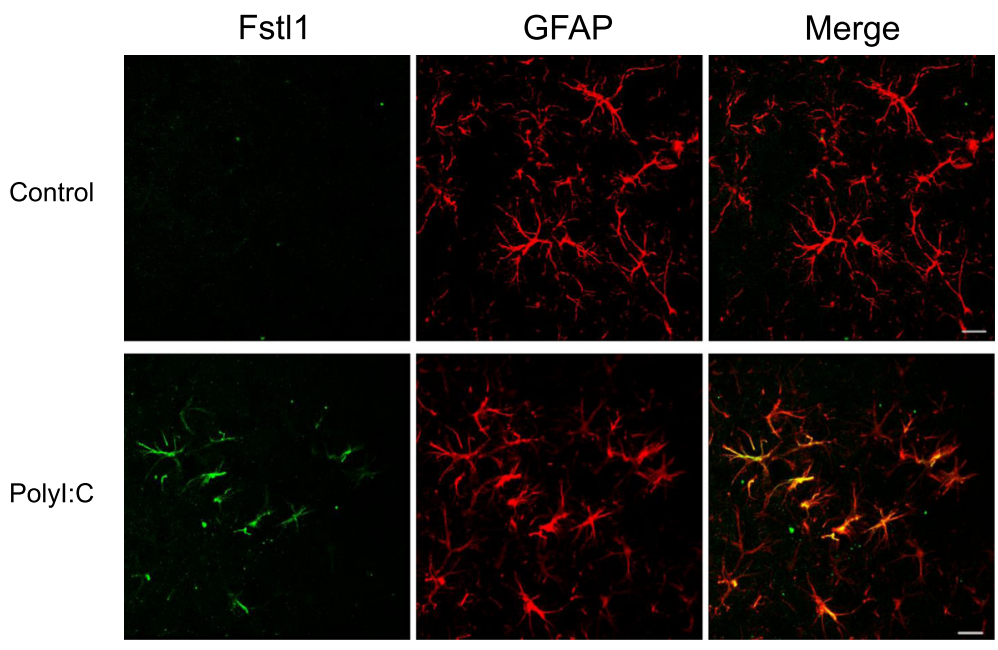

B

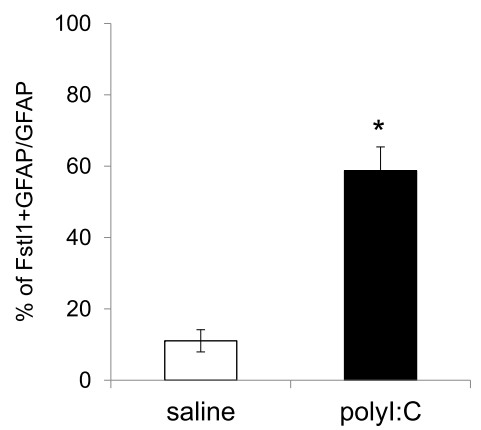

Fig. 5 In vivo expression of Fstl1 protein in PD7 neonates. Neonatal C57BL/6J mice were administered with a daily subcutaneous injection of saline (control) or polyl:C (5 mg/kg) between PD2 and PD6. a Hippocampal sections were isolated $24 \mathrm{~h}$ after the final drug treatment and Fstl1 (green) and GFAP (red, a marker for astrocytes) protein expressions were assessed by IHC. Scale bar, $10 \mu \mathrm{m}$. b Ratio of Fstl1- and GFAP-double positive cells versus GFAP positive cells was quantified. Values indicate the means \pm SE of three independent experiments. ${ }^{*} p<0.05$ versus control

polyI:C-treated astrocytes. We confirmed that Fstl1 is upregulated in polyI:C-treated astrocytes and acts as a humoral factor to impair neurite elongation of cultured neurons. PolyI:C stimulates toll-like receptor 3 (TLR3) signaling, which leads to production of inflammatory cytokines [17, 21], while the expression level of Fstl1 mRNA increases in response to inflammatory cytokines such as IL-1, IL-6, and TNF- $\alpha$ [22, 23]. Therefore, polyI:C may upregulate Fstl1 mRNA levels through the production of inflammatory cytokines.

Fstl1 is a glycoprotein that is secreted in response to inflammatory signals. Lipopolysaccharide (LPS) increases Fstl1 expression [24, 25]. In the present study, we demonstrated that the extracellular level of Fstl1 in cultured astrocytes was increased by polyI:C treatment, but such increase was attenuated in Ifitm $3 \mathrm{KO}$ astrocytes. Moreover, co-expression of Fstl1 and Ifitm3 in COS7 cells significantly increased the extracellular level of Fstl1 compared to the level in COS7 cells only expressing Fstl1 but not Ifitm3. We speculate that polyI:C treatment may increase the extracellular level of Fstl1 through the inhibitory effect of Ifitm 3 of endocytic activity [17]. An alternative explanation is that Ifitm3 may affect membrane vesicle trafficking and secretion in astrocytes. Because neither the mRNA expression level nor were the intracellular protein level of Fstl1 affected by Ifitm3 expression, regulation of Fstl1 expression may be independent on Ifitm3 expression. Ifitm3-independent expression of Fstl1 was also demonstrated in vivo. Although we attempted to measure extracellular Fstl1 in mice, it has not been detected yet because of analytical limitation. Further study is needed to directly show that the increased extracellular Fstl1 is responsible for the polyI:C-induced neuronal impairment. 
In addition to astrocytes, microglia are involved in the inflammation-related neuronal impairment [26, 27]. PolyI:C activates both astrocytes and microglia through activation of TLR3 $[28,29]$, which led us to hypothesize that Fstl1 expression would be increased not only in astrocytes but also in microglia, in response to polyI:C stimulation. The expression level of Ifitm3 mRNA was significantly increased by treatment with polyI:C both in cultured astrocytes and in cultured microglia. On the other hand, the expression level of Fstl1 mRNA was very limited in microglia compared to astrocytes, and not increased by polyI:C treatment in microglia. Furthermore, the extracellular Fstl1 protein of cultured microglia could not be detected either in the presence or absence of polyI:C treatment. These results suggest that the induction of Fstl1 in response to TLR3 activation by polyI:C may be a specific event in astrocytes, but not occur in microglia.

How does Fstl1 impair morphologic neuronal development? The role of Fstl1 is controversial because some findings suggest that Fstl1 functions as a pro-inflammation cytokine [24, 30-32], but others suggest a role as an anti-inflammatory cytokines $[25,33,34]$. Fstll protects cells from apoptosis in heat failure through Fstl1 receptor disco-interacting protein 2 (DIP2), which activates the Akt signaling pathway [35, 36]. Some reports suggest that Fstl1 works as a scavenger by sequestering bone morphogenetic protein (Bmp)-4, which results in blockage of BMP signaling during development [37-39]. The addition of both Fstl1 and ACM suppressed neurite outgrowth, but addition of Fstl1 alone did not. Co-treatment of Fstl1 with Bmp-4 unaffected to neurites elongation (Additional file 6: Figure S6). A possible explanation is that secreted Fstl1 from astrocytes may inhibit signals promoting neurite outgrowth BMP-independent manner. In DRG neurons, Fstl1 impairs neurite elongation through activation of $\mathrm{Na}$ /K-ATPase [40]. The similar mechanism might operate in the hippocampal neurons. In this study, we could not address the role of Fstl1 in vivo because of technical limitation; it is hard to manipulate gene expression specifically in astrocytes of neonatal mice. Thus, further studies are needed to disclose the role of Fstl1 in polyI:C-induced neuronal impairment.

The association of Fstl1 with neuropsychiatric disorders is unclear. Recently, a SNP in miR-198, whose expression is mutually exclusive to Fstl1, was found to be associated with schizophrenia [41-43]. We found that the expression of Ifitm3 increased the extracellular level of Fstl1, while other studies reported the increased expression of Ifitm 3 in the brains of schizophrenia patients [13-15]. Taken together with our present findings, it is possible that Fstl1 may play a role in the pathophysiology of psychiatric disorders on downstream of Ifitm3.

\section{Conclusions}

From our findings, we conclude that the extracellular level of Fstl1 is regulated by Ifitm 3 in astrocytes. However, Fstl1 itself may not directly inhibit the dendritic elongation of neurons but interrupt neurite elongation by cooperating with some factors in ACM.

\section{Additional files}

Additional file 1: Figure S1. Validation of Fslt1 knockdown, related to Fig. 3. Culture astrocytes were transfected with siRNA targeting for Fstl1 or control siRNA. Western blotting was performed with indicated antibodies. (PPTX $66 \mathrm{~kb}$ )

Additional file 2: Figure S2. Effect of rFstl1 treatment on neurite branch, related to Fig. 4. Neurons were treated with indicated concentration of rmFstl1 or vehicle. Branched number of neurites was counted. Values indicate the means \pm SE $(n=26-36)$. (PPTX $46 \mathrm{~kb})$

Additional file 3: Figure S3. Effect of rFstl1 treatment on cell viability, related to Fig. 4. Neurons were treated with indicated concentration of rmFstl1 or vehicle. The cell viability of neurons was measured. Values indicate the means \pm SE $(n=3)$. (PPTX $43 \mathrm{~kb})$

Additional file 4: Figure S4. Co-expression of Fstl1 with Ifitm3 in the hippocampus of polyl:C-treated neonatal mice, related to Fig. 5. Hippocampal sections prepared from mice treated with vehicle or polyl:C were immunostained with indicated antibodies. Scale bar, $20 \mu \mathrm{m}$. (PPTX $470 \mathrm{~kb}$ )

Additional file 5: Figure S5. Expression of Fstl1 in polyl:C-treated Ifitm3 $\mathrm{KO}$ mice, related to Fig. 5. Hippocampal brain slices prepared from vehicleor polyl:C-treated Ifitm3 KO mice were immunostained with indicated antibodies. Scale bar, 20 m. (PPTX 23832 kb)

Additional file 6: Figure S6. Combinatory treatment of rBmp4 with rFstl1, related to Fig. 4. Neurons were cultured for 5 days (DIV2-7) with culture medium supplemented with the indicated concentration of rmFstl1 and/or rBmp-4. MAP2-positive dendrite length of neurons was measured. Values indicate the means \pm SE of three independent experiments. Scale bar, 50 нm. (PPTX 120 kb)

\section{Abbreviations}

ACM: Astrocyte conditioned medium; Bmp-4: Bone morphogenetic protein-4; CNS: Central nervous system; DIP2: Disco-interacting protein 2; Fstl1: Follistatinlike 1; GFAP: Glial fibrillary acidic protein; Ifitm3: Interferon-induced transmembrane protein 3; IHC: Immunohistochemistry; IL-6: Interleukin-6; LPS: Lipopolysaccharide; MCM: Microglia-conditioned medium; Mmp3: Matrix metalloproteinase 3; Polyl:C: Polyriboinosinic-polyribocytidylic acid; TLR3: Tolllike receptor 3; TNF-a: Tumor necrosis factor-a

\section{Acknowledgements}

We thank Dr. Ulrike C. Lange, David J. Adams, and Azim Surani for providing the Ifitm3 KO mouse. We also thank all members of Yamada Lab for their helpful discussions and support of this work.

\section{Funding}

This work was supported by the Japan Society for the Promotion of Science (JSPS) KAKENHI (Grant-in Aid for Scientific Research (B) $17 \mathrm{H04031}$ to K.Y., $17 \mathrm{H} 04252$ to T. Nabeshima, $17 \mathrm{H} 02220$ to T. Nagai, Grant-in Aid for Challenging Exploratory Research 16K15201 to K.Y., 17K19483 to T. Nagai, Grant-in Aid for Scientific Research on Innovative Areas 24111518 to K.Y., Grant-in Aid for Young Scientists (B) $16 \mathrm{~K} 21080$ to N.I.) and the Private University Research Branding Project from the Ministry of Education, Culture, Sports, Science, and Technology (MEXT), the Astellas Foundation for Research on Metabolic Disorders, The Hori Science and Arts Foundation, Grant for Biomedical Research from Smoking Research Foundation (SRF), the Uehara Memorial Foundation and partially supported by the Strategic Research Program for Brain Sciences, and Research on Regulatory Science of Pharmaceuticals and Medical Devices from Japan Agency for Medical Research and Development, AMED. The Pharmacological Research Foundation, Tokyo. 


\section{Availability of data and materials}

The dataset used and analyzed during the current study are available from the corresponding author on reasonable request.

\section{Authors' contributions}

SY, TNagai, NI, and KY designed the study. SY, TNagai, and NI performed experiments and analyzed the data. SY, TNagai, NI, TNakai, DI, AN, TNabeshima, and $\mathrm{KY}$ analyzed and interpreted the data. SY, NI, TNagai, TNabeshima, and KY wrote the manuscript. All of the authors discussed the results and agreed on the content of the manuscript.

\section{Ethics approval}

This study was approved by the Institutional Animal Care and Use Committee of Nagoya University (Approval No. 30248). Animals were handled in accordance with the guidelines established by the Institutional Animal Care and Use Committee of Nagoya University.

\section{Consent for publication}

Not applicable

\section{Competing interests}

The authors declare that they have no competing interests.

\section{Publisher's Note}

Springer Nature remains neutral with regard to jurisdictional claims in published maps and institutional affiliations.

\section{Author details}

'Department of Neuropsychopharmacology and Hospital Pharmacy, Nagoya University Graduate School of Medicine, 65 Turumai-cho, Showa-ku, Nagoya, Aichi 466-8560, Japan. ${ }^{2}$ Department of Chemical Pharmacology, Faculty of Pharmaceutical Science, Meijo University, 150 Yagotoyama, Tenpaku-ku, Nagoya, Japan. ${ }^{3}$ Faculty of Agriculture and Life Science, Hirosaki University, 3 Bunkyo-cho, Hirosaki, Aomori 036-8561, Japan. ${ }^{4}$ Advanced Diagnostic System Research Laboratory, Fujita Health University, Graduate School of Health Science and Aino University, 1-98 Dengakugakubo, Kutsukake-cho, Toyoake, Aichi 470-1192, Japan.

Received: 7 June 2018 Accepted: 12 October 2018

Published online: 22 October 2018

\section{References}

1. Luo Y, He H, Zhang M, Huang X, Zhang J, Zhou Y, Liu X, Fan N. Elevated serum levels of TNF-alpha, IL-6 and IL-18 in chronic schizophrenic patients. Schizophr Res. 2014;159:556-7.

2. Stojanovic A, Martorell L, Montalvo I, Ortega L, Monseny R, Vilella E, Labad J. Increased serum interleukin-6 levels in early stages of psychosis: associations with at-risk mental states and the severity of psychotic symptoms. Psychoneuroendocrinology. 2014;41:23-32.

3. Schmidt FM, Schroder T, Kirkby KC, Sander C, Suslow T, Holdt LM, Teupser D, Hegerl U, Himmerich H. Pro- and anti-inflammatory cytokines, but not CRP, are inversely correlated with severity and symptoms of major depression. Psychiatry Res. 2016;239:85-91.

4. Brown AS, Begg MD, Gravenstein $S$, Schaefer CA, Wyatt RJ, Bresnahan M, Babulas VP, Susser ES. Serologic evidence of prenatal influenza in the etiology of schizophrenia. Arch Gen Psychiatry. 2004;61:774-80.

5. Khandaker GM, Zimbron J, Dalman C, Lewis G, Jones PB. Childhood infection and adult schizophrenia: a meta-analysis of population-based studies. Schizophr Res. 2012;139:161-8.

6. Khandaker GM, Zimbron J, Lewis G, Jones PB. Prenatal maternal infection, neurodevelopment and adult schizophrenia: a systematic review of population-based studies. Psychol Med. 2013;43:239-57.

7. Ibi D, Yamada K. Therapeutic targets for neurodevelopmental disorders emerging from animal models with perinatal immune activation. Int J Mol Sci. 2015;16:28218-29.

8. Koyama Y. Functional alterations of astrocytes in mental disorders: pharmacological significance as a drug target. Front Cell Neurosci. 2015;9:261.

9. Bailey CC, Zhong G, Huang IC, Farzan M. IFITM-family proteins: the cell's first line of antiviral defense. Annu Rev Virol. 2014;1:261-83.
10. Chesarino NM, MCMichael TM, Yount JS. Regulation of the trafficking and antiviral activity of IFITM3 by post-translational modifications. Future Microbiol. 2014;9:1151-63.

11. Everitt AR, Clare S, Pertel T, John SP, Wash RS, Smith SE, Chin CR, Feeley EM Sims JS, Adams DJ, Wise HM, Kane L, Goulding D, Digard P, Anttila V, Baillie JK, Walsh TS, Hume DA, Palotie A, Xue Y, Colonna V, Tyler-Smith C, Dunning J, Gordon SB, Gen II, Investigators M, Smyth RL, Openshaw PJ, Dougan G, Brass AL, Kellam P. IFITM3 restricts the morbidity and mortality associated with influenza. Nature. 2012;484:519-23.

12. Brass AL, Huang IC, Benita Y, John SP, Krishnan MN, Feeley EM, Ryan BJ, Weyer JL, van der Weyden L, Fikrig E, Adams DJ, Xavier RJ, Farzan M, Elledge SJ. The IFITM proteins mediate cellular resistance to influenza A H1N1 virus, West Nile virus, and denque virus. Cell. 2009:139:1243-54.

13. Hwang Y, Kim J, Shin JY, Kim JI, Seo JS, Webster MJ, Lee D, Kim S. Gene expression profiling by mRNA sequencing reveals increased expression of immune/inflammation-related genes in the hippocampus of individuals with schizophrenia. Transl Psychiatry. 2013;3:e321.

14. Horvath S, Mirnics K. Immune system disturbances in schizophrenia. Biol Psychiatry. 2014;75:316-23.

15. Saetre P, Emilsson L, Axelsson E, Kreuger J, Lindholm E, Jazin E. Inflammationrelated genes up-regulated in schizophrenia brains. BMC Psychiatry. 2007;7:46.

16. Ibi D, Nagai T, Kitahara Y, Mizoguchi H, Koike H, Shiraki A, Takuma K, Kamei H, Noda Y, Nitta A, Nabeshima T, Yoneda Y, Yamada K. Neonatal polyl:C treatment in mice results in schizophrenia-like behavioral and neurochemical abnormalities in adulthood. Neurosci Res. 2009;64:297-305.

17. Ibi D, Nagai T, Nakajima A, Mizoguchi H, Kawase T, Tsuboi D, Kano S, Sato Y, Hayakawa M, Lange UC, Adams DJ, Surani MA, Satoh T, Sawa A, Kaibuchi K, Nabeshima T, Yamada K. Astroglial IFITM3 mediates neuronal impairments following neonatal immune challenge in mice. Glia. 2013;61:679-93.

18. Yamada S, Nagai T, Nakai T, Ibi D, Nakajima A, Yamada K. Matrix metalloproteinase-3 is a possible mediator of neurodevelopmental impairment due to poly: C-induced innate immune activation of astrocytes. Brain Behav Immun. 2014;38:272-82.

19. Lange UC, Adams DJ, Lee C, Barton S, Schneider R, Bradley A, Surani MA. Normal germ line establishment in mice carrying a deletion of the lfitm/ Fragilis gene family cluster. Mol Cell Biol. 2008;28:4688-96.

20. Ibi D, Nagai T, Koike H, Kitahara Y, Mizoguchi H, Niwa M, Jaaro-Peled H, Nitta A, Yoneda Y, Nabeshima T, Sawa A, Yamada K. Combined effect of neonatal immune activation and mutant DISC1 on phenotypic changes in adulthood. Behav Brain Res. 2010;206:32-7.

21. Sobue A, Ito N, Nagai T, Shan W, Hada K, Nakajima A, Murakami Y, Mouri A, Yamamoto Y, Nabeshima T, Saito K, Yamada K. Astroglial major histocompatibility complex class I following immune activation leads to behavioral and neuropathological changes. Glia. 2018;66:1034-52.

22. Clutter SD, Wilson DC, Marinov AD, Hirsch R. Follistatin-like protein 1 promotes arthritis by up-regulating IFN-gamma. J Immunol. 2009;182:234-9.

23. Wilson DC, Marinov AD, Blair HC, Bushnell DS, Thompson SD, Chaly Y, Hirsch R. Follistatin-like protein 1 is a mesenchyme-derived inflammatory protein and may represent a biomarker for systemic-onset juvenile rheumatoid arthritis. Arthritis Rheum. 2010;62:2510-6.

24. Murakami K, Tanaka M, Usui T, Kawabata D, Shiomi A, Iquchi-Hashimoto M, Shimizu M, Yukawa N, Yoshifuji H, Nojima T, Ohmura K, Fujii T, Umehara H, Mimori T. Follistatin-related protein/follistatin-like 1 evokes an innate immune response via CD14 and toll-like receptor 4. FEBS Lett. 2012;586:319-24.

25. Cheng KY, Liu Y, Han YG, Li JK, Jia JL, Chen B, Yao ZX, Nie L, Cheng L. Follistatinlike protein 1 suppressed pro-inflammatory cytokines expression during neuroinflammation induced by lipopolysaccharide. J Mol Histol. 2017;48:63-72.

26. Jha MK, Lee WH, Suk K. Functional polarization of neuroglia: implications in neuroinflammation and neurological disorders. Biochem Pharmacol. 2016;103:1-16.

27. Mondelli V, Vernon AC, Turkheimer F, Dazzan P, Pariante CM. Brain microglia in psychiatric disorders. Lancet Psychiatry. 2017;4:563.

28. van Noort JM, Bsibsi M. Toll-like receptors in the CNS: implications for neurodegeneration and repair. Prog Brain Res. 2009;175:139-48.

29. Ribeiro BM, do Carmo MR, Freire RS, Rocha NF, Borella VC, de Menezes AT, Monte AS, Gomes PX, de Sousa FC, Vale ML, de Lucena DF, Gama CS, Macedo D. Evidences for a progressive microglial activation and increase in iNOS expression in rats submitted to a neurodevelopmental model of schizophrenia: reversal by clozapine. Schizophr Res. 2013;151:12-9.

30. Miyamae T, Marinov AD, Sowders D, Wilson DC, Devlin J, Boudreau R, Robbins $\mathrm{P}$. Hirsch R. Follistatin-like protein-1 is a novel proinflammatory molecule. J Immunol. 2006;177:4758-62. 
31. Ni S, Miao K, Zhou X, Xu N, Li C, Zhu R, Sun R, Wang Y. The involvement of follistatin-like protein 1 in osteoarthritis by elevating NF-kappaB-mediated inflammatory cytokines and enhancing fibroblast like synoviocyte proliferation. Arthritis Res Ther. 2015;17:91.

32. Zhang ZM, Zhang AR, Xu M, Lou J, Qiu WQ. TLR-4/miRNA-32-5p/FSTL1 signaling regulates mycobacterial survival and inflammatory responses in mycobacterium tuberculosis-infected macrophages. Exp Cell Res. 2017;352:313-21.

33. Li D, Wang $Y, X u N$, Wei $Q$, Wu M, Li X, Zheng $P$, Sun $S$, Jin $Y$, Zhang G, Liao $R$, Zhang P. Follistatin-like protein 1 is elevated in systemic autoimmune diseases and correlated with disease activity in patients with rheumatoid arthritis. Arthritis Res Ther. 2011;13:R17.

34. Hayakawa S, Ohashi K, Shibata R, Kataoka Y, Miyabe M, Enomoto T, Joki Y, Shimizu Y, Kambara T, Uemura Y, Yuasa D, Ogawa H, Matsuo K, HiramatsuIto M, van den Hoff MJ, Walsh K, Murohara T, Ouchi N. Cardiac myocytederived follistatin-like 1 prevents renal injury in a subtotal nephrectomy model. J Am Soc Nephrol. 2015;26:636-46.

35. Ouchi N, Asaumi Y, Ohashi K, Higuchi A, Sono-Romanelli S, Oshima Y, Walsh K. DIP2A functions as a FSTL1 receptor. J Biol Chem. 2010;285:7127-34.

36. Tanaka M, Murakami K, Ozaki S, Imura Y, Tong XP, Watanabe T, Sawaki T, Kawanami T, Kawabata D, Fujii T, Usui T, Masaki Y, Fukushima T, Jin ZX Umehara H, Mimori T. DIP2 disco-interacting protein 2 homolog A (Drosophila) is a candidate receptor for follistatin-related protein/follistatin-like 1-analysis of their binding with TGF-beta superfamily proteins. FEBS J. 2010;277:4278-89.

37. Geng Y, Dong Y, Yu M, Zhang L, Yan X, Sun J, Qiao L, Geng H, Nakajima M. Furuichi T, lkegawa S, Gao X, Chen YG, Jiang D, Ning W. Follistatin-like 1 (Fst11) is a bone morphogenetic protein (BMP) 4 signaling antagonist in controlling mouse lung development. Proc Natl Acad Sci U S A. 2011;108:7058-63.

38. Sylva M, Li VS, Buffing AA, van Es JH, van den Born $M$, van der Velden $S$, Gunst Q, Koolstra JH, Moorman AF, Clevers H, van den Hoff MJ. The BMP antagonist follistatin-like 1 is required for skeletal and lung organogenesis. PLoS One. 2011;6:e22616.

39. Xu J, Qi X, Gong J, Yu M, Zhang F, Sha H, Gao X. Fstl1 antagonizes BMP signaling and regulates ureter development. PLoS One. 2012;7:e32554.

40. Li KC, Zhang FX, Li CL, Wang F, Yu MY, Zhong YQ, Zhang KH, Lu YJ, Wang $\mathrm{Q}$, Ma XL, Yao JR, Wang JY, Lin LB, Han M, Zhang YQ, Kuner R, Xiao HS, Bao L, Gao X, Zhang X. Follistatin-like 1 suppresses sensory afferent transmission by activating $\mathrm{Na}$ +, K+-ATPase. Neuron. 2011;69:974-87.

41. Sundaram GM, Common JE, Gopal FE, Srikanta S, Lakshman K, Lunny DP, Lim TC, Tanavde V, Lane EB, Sampath P. 'See-saw' expression of microRNA-198 and FSTL1 from a single transcript in wound healing. Nature. 2013;495:103-6.

42. Hansen $T$, Olsen $L$, Lindow $M$, Jakobsen $K D$, Ullum $H$, Jonsson $E$, Andreassen $O A$, Djurovic S, Melle I, Agartz I, Hall H, Timm S, Wang AG, Werge T. Brain expressed microRNAs implicated in schizophrenia etiology. PLoS One. 2007;2:e873.

43. Beveridge NJ, Cairns MJ. MicroRNA dysregulation in schizophrenia. Neurobiol Dis. 2012;46:263-71.

Ready to submit your research? Choose BMC and benefit from:

- fast, convenient online submission

- thorough peer review by experienced researchers in your field

- rapid publication on acceptance

- support for research data, including large and complex data types

- gold Open Access which fosters wider collaboration and increased citations

- maximum visibility for your research: over $100 \mathrm{M}$ website views per year

At $\mathrm{BMC}$, research is always in progress.

Learn more biomedcentral.com/submissions 